Cinémas

Revue d'études cinématographiques

Journal of Film Studies

\title{
Parcours québécois et canadiens en cinéphonographie
}

\section{Réal La Rochelle}

Volume 3, numéro 1, automne 1992

Cinéma et Musicalité

URI : https://id.erudit.org/iderudit/1001182ar

DOI : https://doi.org/10.7202/1001182ar

Aller au sommaire du numéro

Éditeur(s)

Cinémas

ISSN

1181-6945 (imprimé)

1705-6500 (numérique)

Découvrir la revue

Citer cet article

La Rochelle, R. (1992). Parcours québécois et canadiens en cinéphonographie. Cinémas, 3(1), 95-112. https://doi.org/10.7202/1001182ar

\section{Résumé de l'article}

Depuis Norman McLaren durant les années 40 jusqu’à récemment, la frange expérimentale et exploratoire des cinémas au Québec et au Canada a produit un nombre significatif de films qui sont au coeur de la problématique de la musicalité filmique. Depuis les images el les sons gravés ou peints sur pellicule par McLaren, en passant par le concept d'opéra audiovisuel de Maurice Blackburn et de son Atelier de conception sonore à l'ONF, ou encore par les travaux des Charles Gagnon. Michael Snow, William MacGillivray, Pierre Hébert, les frères Gagné el André Duchesne, entre autres, s'est tissée une tapisserie visuelle et sonore digne de figurer dans le musée imaginaire du nouvel opéra de Michel Fano. 


\title{
Parcours québécois et canadiens en cinéphonographie ${ }^{1}$
}

\section{Réal La Rochelle}

\begin{abstract}
RÉSUMÉ
Depuis Norman McLaren durant les années 40 jusqu'à récemment, la frange expérimentale et exploratoire des cinémas au Québec el au Canada a produit un nombre significatif de films qui sont au coeur de la problématique de la musicalité fïlmique. Depuis les images et les sons gravés ou peints sur pellicule par McLaren, en passant par le concept d'opéra audiovisuel de Maurice Blackburn et de son Atelier de conception sonore à l'ONF, ou encore par les travaux des Charles Gagnon, Michael Snow, William MacGillivray, Pierre Hébert, les frères Gagné et André Duchesne, entre autres, s'est tisséc une tapisserie visuelle et sonore digne de ligurer dans le musée imaginaire du nouvel opéra de Michel Fano.
\end{abstract}

\begin{abstract}
From the work of Norman McLaren in the 1940s until quite recently, the experimental of exploratory fringes of the cinemas of Quebec and Canada have produced a significant number of lilms that are at the heart of the problematic of filmic musicality. From the images and sounds etched or painted on the film by McLaren, through Maurice Blackburn's concept of audiovisual opera and his Sound Conception Workshop at the NFB, or the work of Charles Gagnon, Michael Snow, William MacGillivray, Pierre Hébert, the Gagné brothers and André Duchesne, among others, a tapestry of sight and sound has been woven that is worthy of hanging in the imaginary museum of the new opera by Michel Fano.
\end{abstract}


Cette très fine observation de l'auteur de L'Inhumaine au compositeur belge Hoérée éclaire ce qui fonde la musicalité filmique: le montage.

L'Herbier, au moment où il réfléchit au projet d'un Conservatoire des Arts Nouveaux: Cinéma, Jazz, Radio, Disque, Music-Hall, ne craint pas de confier à un musicien les cours de montage cinématographique ${ }^{2}$. De cette manière, il veut concrétiser la clé de voûte des inventions théoriques et pratiques qui, entre les années 20 et 40, a établi l'apport capital de l'art musical au cinéma.

Cette fusion n'est pas d'abord la présence de la musique dans le film, mais avant tout la dynamique rythmique et structurelle musicale comme fondement du montage filmique, visuel ou audiovisuel, ainsi qu'en ont témoigné, par exemple, Eisenstein et Béla Balazs :

Le tempo du montage n'est pas toujours et seulement respiration et accentuation, le montage n'est pas purement et simplement une sorte de mouvement expressif du contenu dramatique. Le rythme du montage peut acquérir unc valeur propre, indépendante, musicale pour ainsi dire, qui n'a plus qu'un rapport éloigné et irrationnel avec le contenu (Balazs, p. 166).

Dans cette foulée, Balazs aboutit à l'expression : la musique visuelle du montage (p. 166) ${ }^{3}$. Plus explicite encore, Eisenstein, en 1947, dans ses extraordinaires notes «d'un cours sur la musique et la couleur dans Ivan le Terrible», éclaire ces dynamiques filmiques par le recours au phrasé, au legato et à la construction musicale :

En effectuant votre montage, vous devez également assembler et dissocier vos fragments, les combiner jusqu'au moment où votre assemblage «se mettra à chanter» (...) dès l'instant où l'assemblage commence à s'élever jusqu'à la régularité de la construction musicale, on peut dire qu'il commence à être ce qu'il doit être (p. $281)^{4}$.

\section{Du film peint au film-disque}

À l'époque où L'Herbier écrit à Arthur Hoérée, correspond le moment où Norman McLaren vit sa période créatrice consistant à peindre sur pellicule des sons, des musiques et des images. C'est ainsi que le Canada s'ouvrait à son tour à la musicalité filmique, suivi bientôt du Québec. Courant qui ne s'est jamais arrêté depuis, comme en témoigne cette réflexion récente de Jacques Leduc: 
La musıque aun IIım nest pas que la musique aun $111 \mathrm{~m}(\ldots)$. Les voix ont des timbres, les sons ont des couleurs (...) de tous les arts auxquels il peut ressembler, c'est de la musique que le cinéma se rapproche le plus. C'est un art du temps, par opposition aux arts de l'espace (...). Les musiciens entretiennent en commun avec les cinéastes un rapport intrinsèque avec le Temps et une propension à l'organiser de façon onirique (p. 10).

La musicalité filmique, dans les cinémas québécois et canadien, bien que souvent située dans les marges de la production, parfois même en clandestinité, n'en exprime pas moins un esprit de suite (le mot évoque une forme musicale tout autant que la ténacité!), qui est un des traits dynamiques des nouveaux cinémas d'aprèsguerre. Il suffit d'un bref parcours cinéphonographique, la trace de quelques sentiers, pour en éclairer les principales phases, d'abord avec les pionniers McLaren et Maurice Blackburn, ensuite chez ceux de la deuxième génération, comme Michael Snow et Jacques Leduc.

À côté de ces derniers, les années 70 voient se côtoyer, dans l'aura de l'Atelier de conception sonore de l'Office national du film du Canada (ONF), des créations polymorphes où disques et films, bandes sonores et audiovisuelles irradient des images hybrides. Y travaillent des musiciens comme Yves Daoust et Glenn Gould, le cinéaste Louis Portugais ainsi que Charles Gagnon, créateur en arts visuels.

Les années 80, enfin, sont plus riches encore d'un fort éclatement de musiques audiovisuelles, qui sont en même temps feux d'artifice, et qui, à Montréal tout autant qu'à Toronto et à Halifax, ont construit un vaste opéra cinéphonographique. De William MacGillivray à Gilles Groulx, de Peter Mettler à Fernand Bélanger, ou encore avec Pierre Hébert, Serge et Jean Gagné, les cinémas québécois et canadien scintillent de ces ouvrages que Balazs appelait du «cinéma lied», ou encore L'Herbier, des «cinéphonies».

\section{Le primitivisme éclairé des bricolages audiovisuels}

C'est à New York, peu avant d'entrer à l'ONF, que Norman McLaren commença à tracer des éléments visuels et sonores directement sur pellicule $35 \mathrm{~mm}$. Un premier film, Allegro (1939), était acheté par le Guggenheim Museum of Non-Objective Painting et fut suivi de Points et Boucles en 1940.

Le cinéma de McLaren est niché de la sorte d'abord au musée, emplacement paradoxal non seulement par son singulier processus de production (sans caméra ni magnétophone, ni laboratoire), mais par le fait que, comme le souligne Guy Glover, le cinéaste s'installe dans un domaine de la cinématographie «d'où la parole est presque exclue et qui est à la frontière de la musique et de la danse» (p. 9). 
Dans les années 50, McLaren poursuivra dans cette veine avec Voisins, Two Bagatelles, Rythmetic et Jack Paar Credit Titles, pour finir en 1971 avec Synchromie. C'est aussi sur cette route qu'en 1953 puis 1955 McLaren va croiser Maurice Blackburn, lors du tournage de $A$ Phantasy puis de Blinkity Blank. Sur ces films, le compositeur va improviser une partie de sa musique et de sa conception sonore. La musicalité des cinémas québécois et canadien prenait donc racine grâce à ces deux bricoleurs, ces deux «petits génies» à la fois naïfs et rigoureux, espiègles et graves.

McLaren, avec la peinture et la gravure d'images et de sons, s'inventa une musicalité filmique proche de la musique de danse. S'il ne sortit jamais, comme l'a souligné Michel Fano à la rencontre montréalaise Parcours en cinéphonographie (La Rochelle 1992: pp. 36-39), du système synchronique sons/images, c'est, je crois, qu'il a spontanément opté pour une musicalité d'accompagnement et de soutien de la gestuelle et de la visualisation rythmiques, à la manière dont il a souvent travaillé, pour d'autres films, à partir des musiques populaires : jazz et boogie-woogie, marche de Sousa, danses carrées et chansons québécoises, reel de violoneux, orgue de Barbarie, etc.

Ces musiques populaires, souvent exécutées à leur origine sur des instruments simples, parfois même «raboutés», ont donc naturellement inspiré un McLaren qui poussait la logique du bricolage filmique jusqu'à se passer de tout appareil enregistreur. Peindre et graver des sons, tout autant que des images, dans ce contexte, consistait à musicaliser des rythmiques audiovisuelles simples, brutes même, qui se prêtent moins à l'écoute qu'à la participation dansante. McLaren apporta à ses films peints le même soin maniaque et passionné que dans le jazz les musiciens qui exécutent des combinaisons fascinantes de structures modulaires fixes et de plages d'improvisation libre.

Blackburn, à son tour, inspiré à la fois par McLaren et par les possibilités de la musique concrète révélée aux côtés de Pierre Henry et de Pierre Schaeffer, s'éloigna tôt de la composition musicale filmique classique pour plonger dans la dynamique du collage sonore. Il ne rêva plus dès lors à autre chose qu'à réunir ses énergies et son travail dans le projet à l'ONF d'un Atelier de conception sonore, qui deviendrait tout à la fois lieu, studio, fantasme et aboutissement d'un nouvel opéra audiovisuel.

Michel Fano soulignait encore récemment le caractère avantgardiste de cet Atelier, qui ne vécut tout compte fait que sous forme d'esquisse et le temps de quelques films étincelants qui rappellent, durant les années 50 et 60 surtout, les brillances d'étoiles déjà mortes. 
Mais quelles ondes sonores lumineuses, néanmoins, que celles de Blinkity Blank (1955), de Je (1960), de Jour après jour (1962) et de Ciné-crime (1969), qui tissent et tricotent les musiques d'instruments, de boîtes et d'automates avec toutes sortes de sons réels ou patentés, avec toutes sortes de voix et leur généreuse gamme de couleurs, de timbres, de grains.

Défenseur des structures sonores intégrantes, dialectiquement soudées à la bande image, Blackburn a longtemps lutté, pour l'essentiel, en faveur d'une pulsation filmique et d'une composition audiovisuelle capables de rendre les images et les sons indissociables. C'est en tant que musicien qu'il rêvait à la réalisation intégrale d'un film, à la manière dont un McLaren, par ses films peints, avait ajouté le statut de musicien à celui de cinéaste 5 . Si Blackburn n'atteint spécifiquement ce rêve que dans une seule réalisation, Ciné-crime, il a par ailleurs avec McLaren, Louis Portugais et Clément Perron, plus tard avec Anne-Claire Poirier dans Mourir à tue-tête (1979), travaillé en symbiose pour obtenir des cinéphonies modernes, qu'il appelait aussi des «formes musicales audio-visuelles» 6 .

\section{Michael Snow : Eye and Ear Control}

Tout en reconnaissant que ses films expérimentaux ont surtout mis l'accent sur le «vocabulaire visuel, et plus particulièrement sur les mouvements de caméra», l'auteur de New York Eye and Ear Control souligne :

[This film] was my first attempt to deal with what I was starting to see as a fabulous practically uncharted artistic area: that of imagesound relationship in the cinema. Almost all of my subsequent films have been developments in this area (...) (p. 24).

Cinéaste des plus singuliers dans le Canada anglais, grand chaman du cinéma expérimental, Michael Snow détient la particularité d'être aussi (et d'abord) musicien, ce qui lui fait rechercher, comme dans Wavelength, les «dons de la prophétie et de la mémoire que seuls le film et la musique sont capables d'offrir» (p. 21) 7 .

Son avant-dernier film, Seе Yoи Later (1990), témoigne d'une fascinante musicalité filmique. Ce court métrage n'est formé pratiquement que d'un seul mouvement de travelling circulaire, au ralenti extrême, qui suit un employé de bureau à la fin de sa journée de travail. L'employé regarde sa montre, se lève, prend son imper, passe devant le pupitre d'une collègue qu'il salue, se dirige vers la porte et sort.

Ce long cycle visuel, dont les couleurs du décor, des costumes, des accessoires et des éclairages varient subtilement et presque 
imperceptiblement, comme pour produire d'autres cycles plus restreints 8 , est accompagné d'une série de plages sonores qui répètent un noyau musical de base avec peu de modulations à la manière des formes circulaires d'un disque.

Le module sonore de base est introduit par une série de coups/bruits de percussions, de manière linéaire, avant que d'autres sons ne forment plusieurs boucles comme des cercles. Les diverses reprises de ce module, au fur et à mesure que se déploient dans le long travelling circulaire les fragments de gestes et de mouvements, font penser à un disque programmé qui se répète jusqu'à l'épuisement des cycles visuels. Ainsi, cette formule d'une musique spécifiquement phonographique, visualisable mentalement comme les sillons du vinyle, fait corps avec la giration visuelle (quoique de façon asynchronique dans les mouvements), qu'elle supporte et alimente jusqu'à son terme.

L'intérêt de Michael Snow pour la relation son/image, comme il s'en explique à Robert Morgan (pp. 24-26), a commencé tôt, au milieu des années 60, avec New York Eye and Ear Control. La trame musicale de ce film, jouée par des jazzmen, et notamment Albert Ayler, Sonny Murray, Don Cherry et Gary Peacock, fut enregistrée en continuité et sans recours à la bande image, alors en cours de montage. Snow a voulu ainsi juxtaposer deux entités distinctes (comme dans la dualité apollinienne et dionysiaque), qui s'influencent réciproquement, mais sans se submerger l'une l'autre. Ce procédé, encore une fois, témoigne d'une volonté certaine, chez cet artiste, de combiner, sans les détruire, les composantes issues et de sa pratique de cinéaste et de celle de musicien producteur de disques, d'opérer dans le film sonore une profonde démarche cinélphonographique.

Dans Wavelength, explique Snow:

(...) there's a glissando that takes about forty minutes to go from its lowest note to its highest [50 cycles per second to 12,000 c.p.s.]. It happens to be electronically made, and it parallels or is equivalent to the zoom in ear terms. It is a very abstract sound, I'd say, or a concrete sound (Morgan, p. 24).

Dix ans plus tard, dans Rameau's Nephew..., Michael Snow construira une bande sonore davantage basée sur du matériel sonore ambiant synchrone, qui comprend beaucoup de dialogues. Mais le cinéaste, ici, ne veut pas se contenter de la synchonicité «naturaliste» du lip-sync. Il joue sur ce dialogue pré enregistré, en tout sens: distorsions, coupures de certains mots, ajouts de sons étrangers, masques, asynchronisation de phrases, etc.

Il en résulte une distanciation volontaire (forcenée) entre la bande son et l'image, qui fait apparaître les dialogues comme ceux 
d'une langue étrangère, qui serait tellement incompréhensible qu'on ne peut plus, souligne Snow dans une très belle formule, l'écouter comme langage (véhicule de sens informatif) mais comme musique : «There are changes that you can make with the sound which will veer it toward the music side as opposed to the information side» $($ p. 24) .

\section{Le petit théâtre de Jacques Leduc}

Dans la longue et surprenante série Chronique de la vie quotidienne, qu'il réalisa à l'ONF à la fin des années 70, le cinéaste Jacques Leduc eut à deux reprises l'occasion de travailler avec Yves Daoust, compositeur électro-acousticien. D'abord dans l'avant-dernier épisode Samedi. Le ventre de la nuit (1977), puis dans l'épilogue Le Plan sentimental (1978).

Cette collaboration est doublement intéressante, d'abord parce qu'elle marque une des rares expériences entre un membre de l'Atelier de conception sonore et un cinéaste du direct documentaire, en faisant sortir la musique électro-acoustique de la zone du cinéma d'animation où elle tendait à demeurer confinée. Ensuite parce qu'elle permet à Leduc une application concrète de son principe sur la musicalité filmique.

Ces deux films de la Chronique expriment en effet une symbiose d'images et de sons tissés en une pulsation musicale assez complexe, mais toujours juste, dans laquelle l'intervention électro-acoustique s'inscrit naturellement en s'imbriquant dans les voix et les sons, les prolongeant, puis en leur donnant forme et rythme de manière structurante.

L'électro-acoustique trouve de cette façon un terrain fertile où s'exprimer, en poussant jusqu'au bout la logique du cinéma direct où la bande sonore n'est jamais seulement la captation des voix et bruits ambiants, calque de l'image, mais nécessite un travail de choix et de traitements acoustiques, puis de montage et de mixage. Cette donnée concrète des sons directs, Daoust la prend telle quelle puis, imperceptiblement d'abord, et ensuite de manière plus nette, la précise aux plans onirique et abstrait.

Ce travail est plus évident dans Le Plan sentimental, puisque cet épiloque ne contient pas à proprement parler de dialogues d'intervenants en situation, comme dans les sept épisodes précédents. Ce court métrage est une synthèse de l'ensemble de la Chronique. Il comprend quelques images en travellings de rues et de routes, de cours d'écoles, travellings en plans-séquences assez longs, parfois coupés de plans flashes ultra-rapides, qui culminent sur un écran fantomatique au milieu d'un ciné-parc vide. Le film se termine sur une brève séquence animée à partir d'une photo 
polaroïd, qui se transforme en porte qui se referme toute seule en claquant.

La bande sonore de ce film est captivante. Elle puise dans les sons de tournage des bribes de paroles plus ou moins nettes, toutes sortes de bruits, des musiques pré-enregistrées d'ambiance, des comptines, des fragments radiophoniques en anglais ou en français, des hurlements de haut-parleurs de fêtes foraines. Par exemple, dans le plan-séquence émouvant de l'écran du ciné-parc, des sifflements de vent sont mixés à des bribes de bandes sonores venant du projecteur dans la cabine, de brefs extraits opératiques de $O$ Sole mio... Ces éléments sont interreliés, regroupés, rythmés par Yves Daoust, augmentés de répétitions en boucles de certains modules sonores, culminant sur l'écran vide du ciné-parc désert.

Le «petit théâtre» de Jacques Leduc, on le trouve à son meilleur dans Le Ventre de la nuit, où s'expriment avec acuité et nostalgie les fêtes populaires du samedi soir, fêtes foraines et bingos, cinéparcs, petit «show» miteux d'hypnotisme, veillées sur les balcons dans les ruelles; on le trouve par-dessus tout dans la dernière partie du long métrage, au moment de la fête de clôture du cinéma Capitol de Famous Players (rue Ste-Catherine à Montréal), suivie de la démolition du palace.

Après une ouverture musicale à l'orgue de barbarie, le film démarre en un long plan-séquence fixe sur quatre hippies fêtant sur la galerie arrière d'une cour minuscule qui donne sur une ruelle. C'est la veille de la St-Jean Baptiste. Bière, musique western ambiante, cris d'enfants off dans la ruelle, petits spectacles donnés tour à tour par chaque personnage : danse en sabots, danse de la fille sur un reel irlandais, vernissage d'un tableau et d'une photo. Toutes ces scènes, Leduc les cadre en demi-ensemble fixe, dans une position anachronique, comme dans les premiers films enregistrant du théâtre filmé.

Après cette entrée en matière, le cinéaste va relier en montage parallèle des séquences de la foire agricole de St-Hyacinthe (manèges et jeux divers, bingo monstre, marathon de danse du genre «on-achève-bien-les-chevaux»), avec celles d'un vieil homme qui produit des séances d'hypnotisme, de la soirée d'un ciné-parc, ainsi que de la clôture et la démolition du cinéma Capitol. Théâtre/cinéma : operascopes 10 populaires d'un samedi soir, éparpillés dans l'axe Montréal/St-Hyacinthe croisant le ciné-parc en rase campagne.

Dans ce parcours de fin d'après-midi («Quatre heures à l'horloge Coca-Cola», dit une voix off de haut-parleur à la fête foraine), qui passe ensuite au coucher de soleil («sur la ruelle Sunset Boulevard», dit une autre voix off), puis va jusqu'à la nuit («Onze heures à l'horloge Coca-Cola»), la courtepointe du Ventre 
de la nuit est cousue de tous ces morceaux et pièces visuels et sonores d'une longue fête triste et quétaine, où le public est autant acteur que spectateur, où alternent plans fixes et travellings ondulants, étroitesse des tréteaux et délires imaginaires.

Dialectiquement, la bande sonore faufile tous ces morceaux visuels, et non seulement les faits tenir ensemble, mais rythme et structure le temps de la fête, son départ dynamique et fringuant, son crescendo bruyant et tapageur, puis son finale mélancolique et terne, où s'accumulent les désillusions, les pleurs, le silence, les détritus.

Comme dans Le Plan sentimental, ce long métrage mixe un riche ensemble de voix et de bruits dominé par les crachements des micros et haut-parleurs, le bric-à-brac strident de la cabine de projection du ciné-parc, les radios et les magnétophones des voisins... S'en détachent, de façon plus nette, l'orgue de barbarie du début, puis des boucles électro-acoustiques au ciné-parc (sorte de caisse enregistreuse répétitive, bruits des rotations des projecteurs), une brève plage au ralenti ponctuant la fin du marathon de danse, un passage de clavecin électronique, très vif et rapide, après le bingo; et puis, à la fin du film, sur les images de démolition du Capitol, la chanson populaire off qui grince : «Grand'maman, oh! oui grand'maman, vous avez dû passer par là...»

\section{Le cinéma par le détour phonographique}

Quel serait le film québécois le plus bizarre, le plus inattendu, le plus au coeur de la musicalité cinématographique, au surplus réalisé non pas par un cinéaste, mais par un créateur en arts visuels, celui-là même qui a produit en 1986, pour le hall du Cineplex Odeon Le Faubourg à Montréal, la sculpture-peinture «Transition/Illusion/Réflexion»? Au bout de cette question apparaît le court métrage Pierre Mercure de Charles Gagnon, peut-être le seul film-disque à avoir été produit.

D'une durée de 33 minutes $1 / 3$, s'inscrivant par là-même dans le temps phonographique de la vitesse de rotation du vinyle, ce film construit son hommage-épitaphe au musicien Mercure en deux cycles de girations interreliés. À l'image, une courte séquence des funérailles du musicien, filmée volontairement en home movie, reprise inlassablement en modulations de noir \& blanc et de filtres couleurs, en tirages négatif et positif; au niveau sonore, une autre boucle, asynchrone avec l'image, métissage de folklore québécois et d'électro-acoustique, elle aussi «toujours recommencée»...

Exemple extrême, oeuvre-limite, ce «film-disque» n'est pas aussi isolé qu'il n'y paraît au premier abord. Il témoigne à sa façon d'une tendance originale et singulière, dans l'audiovisuel canadien 
et québécois, à lier cinéma et art musical phonographique, et ce, de diverses manières. Michael Snow, cinéaste et musicien, pratique les deux arts séparément, mais aussi, comme on l'a vu, en les nouant musicalement dans ses films.

Il y a des exemples plus surprenants encore. Au premier chef celui de Glenn Gould, qu'on retrouve sur ce terrain non comme pianiste, ni non plus comme musicien au cinéma - où il a assez peu travaillé - mais comme réalisateur de documentaires radiophoniques. Et c'est justement dans ces créations phonographiques pour la radio que Glenn Gould s'empare paradoxalement d'une technico-esthétique filmique. Au surplus, il y emprunte la musicalité filmique même, puisque dans ses «drama documentaries» sonores, dans son art phonographique contrapuntique, c'est au langage filmique prégnant de musique que le musicien se refère.

Radio as Music, dit le titre du reportage vidéo, tourné au moment où Gould procède au mixage de Quiet in the Land avec l'ingénieur du son Donald Logan. "Radio as Cinema», pourrait-on ajouter du même souffle, en faisant la synthèse de ces oeuvres phonographiques de Gould par métissage cinéma/musique.

\footnotetext{
John Thompson. - You relate all your audio problems with video terminology...

Glenn Gould. - Yes that's right.

John Thompson. - Are you a frustrated movie director?

Glenn Gould. - There is a strong visual component in radio...11
}

Plus tard, en 1980, dans A Glenn Gould Fantasy, le musicienréalisateur répète son attachement au cinéma. Il se refère encore au «filmic background» de ses créations radio, il définit la monophonie de The Idea of North comme du cinéma en noir \& blanc. Plus encore, Gould n'arrêtera jamais de puiser dans le vocabulaire et la fabrication filmiques des fondus et fondus enchaînés, des montages contrapuntiques ou polyphoniques, des mixages de voix, de bruits et de musiques. Et c'est de la sorte qu'il léguera un fabuleux héritage de «cinéphonies» phonographiques: d'abord, son immense Solitude Trilogy, puis ses «films» musicaux radiophoniques sur Casals, Schoenberg, Stokowski et Richard Strauss, sans oublier cette éblouissante Fantasy pour l'album commémoratif des disques CBS 12 .

Le détour phonographique ne distraira jamais Glenn Gould de nicher au coeur du cinéma, d'un cinéma qui, privé de son compagnon visuel, n'en révélera que mieux son essentielle musicalité. Mais il ne fut pas seul, et les années 70 furent particulièrement créatrices sur ce terrain. Si Charles Gagnon produit dans l'isolement son film-disque Pierre Mercure, ailleurs 
c'est du côté de l'Atelier de conception sonore de l'ONF qu'il faut chercher le cinéma phonographique.

Y gravitent, dans un certain désordre, mais animé de la même passion dont brûlait un Blackburn depuis 20 ans, des créateurs comme Anne-Claire Poirier, Jacques Leduc, Alain Clavier et Yves Daoust. Certains fabriquent des bandes sonores filmiques qu'ils font éditer en disques (Musiques de l'ONF) ou, plus simplement, qu'ils créent directement en produits phonographiques. Alain Clavier signe un disque sur La Qualité de la vie; le cinéaste Louis Portugais, qui avait réalisé Je avec Maurice Blackburn, se commet à son tour, avec Jocelyne Archambault, dans un hörspiel sur La Radio communautaire (resté inédit).

Ces rares productions, comme des satellites en errance, suivent l'esprit gouldien de la musicalité filmique, en s'inscrivant dans l'aura internationale des hörspiel qui avaient séduit Brecht et Walter Ruttmann en Allemagne, Pierre Schaeffer en France, ou encore Bernard Herrmann et Orson Welles aux USA - les deux complices faisant le pont entre la radio du Mercury Theatre à New York et le cinéma à Hollywood.

Ce que ces «musiciens audiovisuels» québécois et canadiens apportaient à ce courant, au sein de l'éruption du cinéma direct, était la volonté de lier le son du direct à la logique de l'art phonographique, plutôt qu'aux mirages du «son-vérité» et au fauxsemblant naturaliste de la synchronicité audiovisuelle. Ils réalisaient ainsi l'embryon de ce qu'aujourd'hui un musicien électro-acousticien comme Robert Normandeau appelle le «cinéma pour l'oreille», et les concepteurs sonores filmiques des «compositions» ou des «partitions» pour les films.

Au milieu des années 80, Yves Daoust devait, dans son Hommage à Maurice Blackburn pour la radio mf de Radio-Canada, illustrer magnifiquement ces détours phonographiques qui témoignent des pulsations et des constructions musicales filmiques.

\section{Vent d'est}

La seconde moitié des années 80 a donné naissance, au Canada anglais, à une nouvelle couvée de cinéastes dont le commun dénominateur, la constante sont l'hypersensibilité à la bande sonore et à sa musicalité contrapuntique avec l'image. Ces cinéastes ont fait ainsi éclater, dans l'expression postmoderne, ce qui était encore à l'état underground dans les décennies précédentes, et ce dans une gamme très large de films: l'expérimental, le documentaire-essai, le long métrage de fiction.

Ainsi vont, par exemple, à Vancouver The Traveller (1989) de Bruno Lazaro Pacheco, comme à Toronto les joyaux de Patricia Rozema, d'Atom Egoyan et de Peter Mettler. Mais un corpus, plus 
étonnant encore peut-être, se trouve à l'extrême est du pays, à Halifax, aux pieds de l'Atlantique, dans une solitude magnifique, inattendue, à l'image du Latecomers de Glenn Gould, où «la mer sert de basse continue» (Guertin, p. 193). Il s'agit de l'oeuvre de William MacGillivray qui, depuis l'étonnant Life Classes (1987), a produit coup sur coup: I Will Not Make Any More Boring Art (1988), The Vacant Lot (1989), Understanding Bliss (1990).

MacGillivray, outre la scénarisation et la réalisation, s'occupe de près des montages de ses films (musiques, images), et il a un flair très aiguisé pour leur environnement sonore, pour des entrelacs très nuancés de voix, de musiques populaires et de bruitages.

Cette manière est particulièrement subtile dans son essai I Will Not..., sur le centenaire du Nova Scotia College of Art and Design, pour lequel il confie la musique à Michael Snow, qui y dirige le Canadian Creative Music Collective (CCMC). Fascinante rencontre, au générique d'un même film, entre Snow et MacGillivray, qui ne peut que décupler les pouvoirs de la musicalité filmique. Par exemple, l'ouverture de ce long métrage/essai nous fait plonger au coeur même de la paraphrase de la modernité, quand la musique actuelle jazzée du CCMC est mixée de voix féminines et d'interviews avec Garry Neill Kennedy racontant comment, après 1967, le Centre d'art de la Nouvelle Écosse opéra sa rupture avec la tradition, s'ouvrit à l'art moderne international, et devint unique en son genre en Amérique du Nord.

Après coup, sont tissées de cette manière les multiples séquences où se succèdent les célèbres «visiting artists» du Centre (Les Levine, Krzysztof Wodiczko, June Leaf, Eric Fischi, entre autres), des fragments d'opéras-vidéos de Joseph Beuys et de Nam June Paik, du Coventry de Klaus Von Bruch et des Larmes d'acier de Marie-Jo Lafontaine. Sans compter, en finale, Michael Snow live, recevant un doctorat honorifique et déclarant à l'assistance, sourire en coin : «Congratulation for those of you who are graduating in ambiguity!», avant de se fondre sur la bande sonore, dans la dernière pièce musicale du CCMC. $\mathrm{Si}$, comme le souligne Kennegy un peu avant Snow, plus personne aujourd'hui ne peut croire qu'il y ait une solution à un projet d'éducation artistique, $I$ Will Not Make Any More Boring Art témoigne avec éclat, de manière grave mais sans «nostalgisme», que l'art actuel est celui de l'ère de l'ambiguïté, donc fortement éclaté, sémantiquement plus musical que verbal.

Les moments les plus émouvants des longs métrages de fiction de MacGillivray sont d'ailleurs construits sur de telles plages musicales audiovisuelles ${ }^{13}$. L'ouverture et la coda de Life Classes, par exemple, lents travellings au ralenti mixés de chanson en gaélique, de voix féminines et de crépitements électroniques; 
l'ouverture aussi d'Understanding Bliss, sur les fragments poétiques de la nouvelle de Katherine Mansfield; ou encore ces beaux passages de Vacant Lot: mixages de musique rock et de bruits d'atterrissage d'avion, plus tard les voix du père et de sa fille en musique country évocatrice de "promised land», enfin plus près du finale, les métissages de vents d'hiver, de chant choral, d'avion en décollage et de cordes rock.

\section{Une gerbe de passiflores}

En réalisant en 1985 leur Passiflora, Fernand Bélanger et Dagmar Guessaz Teufel donnaient un des essais filmiques les plus percutants du cinéma québécois. Avec la complicité du concepteur sonore Claude Beaugrand et des musiciens René Lussier, Jean Derome et André Duchesne, à cause aussi du fait que Bélanger est monteur, sonore autant que visuel, Passiflora s'est développé en véritable opéra, genre à peu près inexistant dans cette cinématographie nationale, si on excepte le Gilles Groulx du début des années 80, Au Pays de Zom ${ }^{14}$.

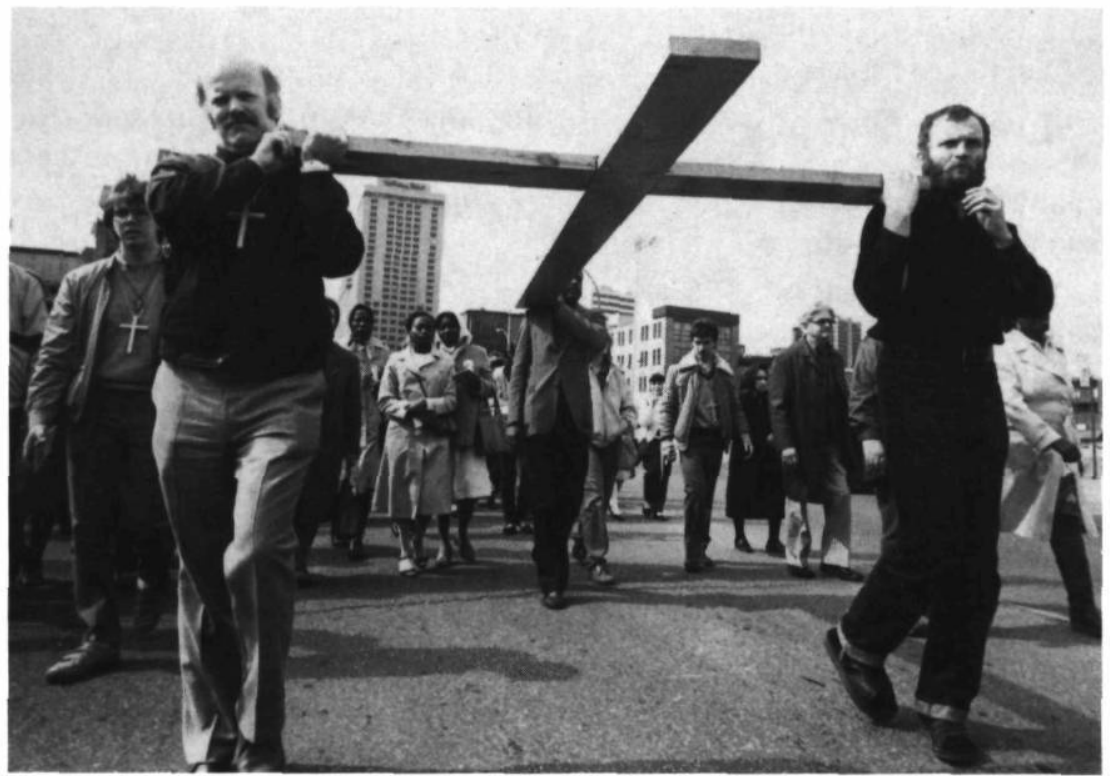

Passiflora de Fernand Bélanger et Dagmar Gueissaz-Teufel (1985) Coll. ONF 
Ces deux films ont une autre parenté : ils utilisent la forme opératique pour parler de l'opéra lui-même, plus précisément de son inscription dans l'audiovisuel contemporain, de sa métamorphose technologique. Car l'opéra, depuis son extinction au théâtre, au début du siècle, s'est régénéré dans et par le cinéma et la vidéo, en y trouvant un nouveau lieu et des techniques d'expression fraîches. Dans ce contexte, pareils opéras technologiques conduisent au coeur même de la musicalité filmique du lyrique.

Comme les «fleurs de la Passion" - les passifloras à figure de Vendredi-Saint kitsch et sanguinolant - ces deux filmopéras au baroquisme tortueux utilisent des codes filmiques lents et hiératiques permettant l'épanchement de grands discours pathétiques contemporains. Pathétiques et fortement médiatisés : celui de l'homme d'affaires québécois Zom, l'autre de Jean-Paul II au Stade Olympique de Montréal.

Au Pays de Zom confie à l'interprète/chanteur Joseph Rouleau trois niveaux de discours musicaux pour exprimer la déprime du grand bourgeois : le parler grandiloquent en légère dissonance, ensuite le parlar cantando, qui s'enchaîne pour finir avec l'aria proprement dit. La musique de Jacques Hétu règle la partition de ces strates vocales, qui se structurent dans les plans-séquences très lents de Gilles Groulx.

Dans Passiflora, la musique est formée d'amalgames de sons traités par Claude Beaugrand (bruits et voix en provenance des prises en direct), imbriqués dans les partitions «bruitistes» de ces tenants de musique actuelle que sont les Lussier, Derome, Duchesne. Cette trame sonore, de la sorte, participe à la fictionnalisation du reportage, à la mise en essai critique du compte-rendu d'événements médiatiques puissants sur le pape et Michael Jackson. La musicalisation filmique nourrit le second degré de ce qui s'appelait le documentaire, et participe à la destruction de ce faux «genre».

Et Passiflora n'est pas seul de son espèce. On retrouve des paramètres opératiques semblables dans Le Royaume ou l'asile (1989), dont la musicalisation audiovisuelle est assumée par un audacieux quatuor : le compositeur André Duchesne, le concepteur sonore Claude Beaugrand, les cinéastes Jean et Serge Gagné. Les collages visuels et sonores, ici, laissent s'entrechoquer des ondes multiformes issues des arts plastiques, du théâtre, de reportages radio, d'interprétations musicales, de docu-drames, des décors naturels de la Côte-Nord, etc.

Par ailleurs, ces dernières années, deux cinéastes participent passionnément au cinéma de la musicalité structurante. Pierre Hébert à Montréal, au Studio français d'animation de l'ONF, Peter 
Mettler à Toronto comme indépendant, de film en film, de fil en aiguille, construisent des oeuvres dont les trajectoires sont imperturbablement liées à la musique, voire fondées sur elle.

Comme ces deux cinéastes se sont vu offrir de participer à cette livraison spéciale sur «musicalité/cinéma», il sied donc de les laisser s'exprimer sur leurs pratiques et sur leurs fondements esthétiques et théoriques. Pour ma part, j'ai eu l'occasion de décrire les rythmes de «défenestration audiovisuelle» propres aux performances musique/cinéma de Pierre Hébert depuis près de 10 ans; je ne peux que répéter ici comment ce cinéaste, «avec une fougue, une fièvre, un dynamisme singulier, a entrepris de se tenir en position hors-cadre, de voyager en contrebande (...) par une pratique sans précédent dans le cinéma québécois et à l'échelle internationale» (1990 : p. 191),dans laquelle dialoguent en mode live les jeux de musiciens et la gravure sur pellicule. Hébert se livre à une sorte de danse audiovisuelle dans laquelle, au corps performant, est substitué l'écran de traits et d'images rythmés, produits à chaud.

Pour sa part, Peter Mettler croit intensément à la musique comme forme structurante d'expression filmique. Dans Scissere, Eastern Avenue et The Top of His Head, le cinéaste fabrique des montages et des mixages musicaux à partir de sons de tournages, de sons additionnels et de musiques pré enregistrées ou live. Si Fred Frith est en performance dans The Top of His Head, Eastern Avenue se sert d'improvisations sur images des musiciens Christie MacFadyen et Joey Hardin, de même que de fragments phonographiques par Jean-Marc Larivière, Harmonic Choir Celestial Harmonies et Fred Frith. Pour Scissere, Mettler emprunte aux disques de Meredith Monk, Max Roach, Ornette Coleman, Bruno Degazio, de chants grégoriens et du Ramayana Monkey Chant. La musicalité filmique est si prégnante chez Mettler, qu'à un moment donné de nos conversations et échanges de notes sur ses films, je me suis cru justifié de lui demander : «Tout compte fait, Peter Mettler, êtes-vous un cinéaste ou un musicien audiovisuel?» La question n'a pas paru incongrue au cinéaste, pour qui la musique permet l'expression audiovisuelle dans l'au-delà de la logique et du verbe signifiant. Quel compositeur disait que la musique, c'est ce que la parole n'arrive plus à exprimer?

Au cinéma, la parole n'est pas seulement celle des personnages ou des témoins, c'est encore celle de la logique et du réalisme des images, ou bien des synchronicités «naturelles». La musique filmique, extra verbale, celle qu'on peut appeler audiovisuelle, détruit ou contourne la sémantique des discours visuels et sonores, pour chercher des significations différentes dans les rythmiques et 
les structures de montage, dans l'entrelacement des diverses couches de mixage.

Cette quête incessante de la musicalité filmique est inscrite dans la genèse du cinéma, et se manifeste tôt comme une des relèves de la musique occidentale, dont l'évolution au début du XXe siècle

entre dans une sorte de goulot d'étranglement. J'aime de temps en temps rappeler qu'un des derniers chefs-d'oeuvre de cette évolution, le Pelléas et Mélisande de Debussy, en 1902, est créé surprenante coincidence - au moment de l'arrivée du cinématographe. On disait alors : «Que peut-on faire après Pelléas?» La réponse apparaît peut-être maintenant un peu plus claire : «Après Pelléas, eh bien! on fait du cinéma, de la musique filmique...»

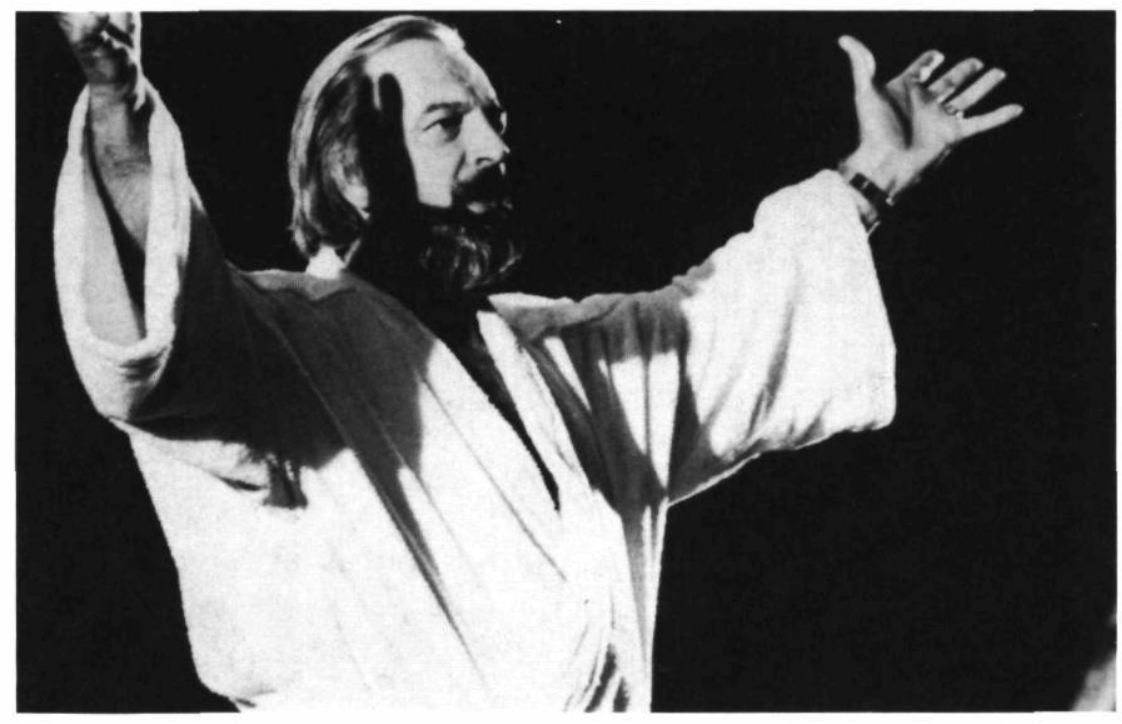

Au pays de Zom de Gilles Groulx (1982)

Photo : Bernard Fougères. Coll. Cinémathèque québécoise

Quelques cinéastes québécois et canadiens, à leur heure et avec leurs moyens, toujours avec la même passion, ont décidé de puiser dans la musique l'énergie de leur cinéma «post-Pelléas»... 


\section{NOTES}

1 Extrait d'un ouvrage à paraître, Parcours nord-américains en cinéphonographie. "Cinéphonographie» : néologisme archaïsant formé à partir des noms des inventions d'Edison pour synchroniser l'image filmique et le son musical : le «Kineto-phonograph» en 1895 et le «Cinephonograph» en 1911.

2 Cette lettre de L'Herbier est citée par Louise Cloutier, dans son mémoire de maîtrise en musicologic : Arthur Hoérée, musicien d'écran, technicien du cinéma et critique cinématographique (Paris : Sorbonne, 1977) p. 19. C'est dans le même ouvrage qu'est citéc aussi l'appellation «cinéphonies» (p. 43), qu'utilisait L'Herbier pour décrire des films musicaux.

3 Dans L'Esprit du cinéma. C'est dans son autre ouvrage, Le Cinéma. Nature et évolution d'un art nouveau (Paris : Payot, 1979), que Béla Balazs utilise les expressions «cinéma lyrique» et «cinéma lied» (p. 229). À remarquer que pour Eisenstein autant que pour Balazs, la musicalité filmique existe dès le cinéma «muet». Balazs, dans Le Cinéma, parle de la «musique des images muettes" (p. 229).

4 Dans $\mathrm{Au}$-delà des étoiles. Eisenstein précise bien par ailleurs (p. 274) que la musicalité filmique n'est pas celle qui «se fait directement dans la musique» d'un film, mais celle qui forme la structure rythmique et le dessin musical interne du film. Il ajoute enfin, pour lever toute ambiguïté : «Dans le cinéma audiovisuel, la musique commence à partir de l'instant où la coexistence ordinaire du son et de l'image cède la place à une association arbitraire du son et de l'image, c'est-à-dire à partir de l'instant où le synchronisme naturel cesse d'exister» (p. 282).

5 McLaren fut désigné musicien de cinéma dans Helmut Kallmann, Gilles Potvin et Kenneth Winters (dir.), L'Encyclopédie de la musique au Canada (Montréal : Fides, 1983).

6 En rappel de son ouvrage fait en collaboration avec Marthe Blackburn et Norman McLaren: Jeunesses musicales du Canada, Six formes musicales audiovisuelles (Montréal, 1967). Ouvrage illustré accompagné d'un disque.

7 «(...) to utilize the gifts of both prophecy and memory which only film and music have to offer.»

8 Dans le même ouvrage (p. 23), Snow parle de l'expérience, dans $L a$ Région centrale (1971), où se combinent des cycles dans les cycles: «There are circles within circles and cycles within cycles.»

9 Le titre intégral du film est Rameau's Nephew by Diderot (Thanx to Dennis Young) by Wilma Schoen. Sur le travail audiovisuel de Snow, voir aussi Michel Larouche, «Vers la symbiose image-son dans le cinéma expérimental: une utopie?», Protée vol. 13, no 2, été 1985. Il existe par ailleurs des analyses importantes du travail filmique de Snow dans R. Bruce Elder, Image and Identity. Reflections on Canadian Film and Culture (Wilfrid Laurier University Press, 1989).

10 «Operascope»: nom donné, au début du siècle, à de petits cinémas populaires, aux États-Unis et au Canada, avant l'arrivée des grands palaces.

11 Radio as Music, réalisation de John Thompson, Toronto, CBC, 1975.

12 Voir Glenn Gould pluriel, textes réunis par Ghyslaine Guertin, Montréal : Louise Courteau éd., 1988. The Solitude Trilogy comprend : The Idea of North (1967), The Latecomers (1969), The Quiet in the Land (1977) [voir analyse de John P.L. Roberts, p. 169]. Les documentaires contrapuntiques englobent pour leur part: Leopold Stokowski: a Portrait for Radio (1971); Pablo Casals: a Portrait for Radio (1973); Schoenberg: the First Hundred Years. Fantasy- 
Documentary (1974); Richard Strauss: the Bourgeois-Hero (1979) [voir Thérèse Sabriat, p. 198]. Finalement, «A Glenn Gould Fantasy», dans The Glenn Gould Silver Jubilee Album, CBS M2X 35914, 1980.

13 Pierre Véronneau parle de l'importance des «ouvertures» filmiques chez MacGillivray, dans «À l'Est, rien de nouveau??», Renaissance du cinéma d'auteur canadien-anglais (Montréal . Cinémathèque québécoise, 1991).

14 Voir Réal La Rochelle, "Gilles Groulx. "Collager" politiquement le culturel québécois», Copie Zéro no 20 (mai 1984).

\section{OUVRAGES CITÉS}

Balazs, Béla. L'Esprit du cinéma. Paris : Payot, 1977.

Eisenstein. Au-delà des étoiles. Paris : Cahiers du cinéma - 10/18, 1974.

Glover, Guy. «Avant-propos». McLaren. Montréal : Office national du film du Canada, 1980.

Guertin, Ghyslaine. Glenn Gould pluriel. Textes réunis. Montréal : Louise Courteau éd., 1988.

La Rochelle, Réal. «Entretien avec Michel Fano». 24 Images 60 (printemps 1992) pp. 36-39.

La Rochelle, Réal. «Les Performances cinéma/musique avec Pierre Hébert. La Défenestration audiovisuclle (notes pour un essai)». Canadian Journal of Political and Social Theory vol. 14, nos 1-3 (1990) pp. 190-203.

Leduc, Jacques. "Notes, en suite». La Revue de la Cinémathèque 5 (fév.-marsavril 1990).

Morgan, Robert. «Playing With Time : Interview with Michael Snow». Ear: Magazine of New Music vol. 9 no $5 /$ vol. 10 no 1 (Fall 1985) pp. 24-26.

Snow, Michael. Michael Snow. Rétrospective. Montréal: La Cinémathèque québécoise, 1975. 